\title{
Unruptured sinus of valsalva dissecting into interventricular septum: a windsock in the heart
}

\begin{abstract}
Sinus of valsalva aneurysm is a rare cardiac anomaly and is usually silent clinically for a long time until and unless it becomes large and compresses or dissects adjacent structures or ruptures. Here we present a young gentleman with history of breathlessness and two episodes of syncope for 5-6months. TTE and TEE confirmed the diagnosis. He successfully underwent surgical repair and was asymptomatic during follow up.
\end{abstract}

Keywords: echocardiography, sinus of valsalva aneurysm, dissection into IVS

\author{
Volume 8 Issue 6 - 2017 \\ Vinod Kumar, Ravinder Singh Sambi, Rekha \\ Mishra, Vinod Sharma \\ Department of Cardiology, National Heart Institute, India
}

\begin{abstract}
Correspondence: Rekha Mishra, Department of Cardiology, National Heart Institute, 49-50, Community Centre, East of Kailash, New Delhi- I I0065, India
\end{abstract}

Received: October 18,2016 | Published: May 12, 2017

\section{Introduction}

Sinus of Valsalva aneurysms (SVA) are rare cardiac anomalies, which may be acquired or congenital, most commonly involving the right coronary sinus in $80-85 \%$ cases, non coronary sinus or rarely left coronary sinus. ${ }^{1}$ A congenital SVA is usually clinically silent but may vary from a mild, asymptomatic dilatation detected in routine 2-dimensional (2D) echocardiography to symptomatic presentations related to the compression of adjacent structures or intracardiac shunting caused by rupture of the SVA into the right side of the heart. ${ }^{2}$ Aneurysms of sinus of valsalva and coexisting congenital heart diseases like Ventricular septal defect (VSD), Aortic Regurgitation (AR), etc can easily be diagnosed with 2D/3D transthoracic (TTE) or transesophageal (TEE) echocardiography. Early diagnosis and immediate surgical management can reduce the morbidity and mortality in most cases. A congenital SVA is more common and most often caused by weakness at the junction of the aortic media and the annulus fibrosus. ${ }^{3}$ An acquired Sinus of valsalva aneurysm is caused by secondary degeneration of the elastic connective tissue that occurs from atherosclerosis or infection (bacterial endocarditis, syphilis, and tuberculosis), trauma, systemic inflammatory diseases (Behçet's disease, ankylosing spondylitis) and connective tissue disorders (systemic lupus erythematosus, Marfan syndrome). ${ }^{4}$ Unruptured SVAs usually remain asymptomatic, but sometimes can cause cardiac rhythm abnormalities, myocardial ischemia, and systemic embolic events. ${ }^{5}$ Diagnosis of sinus of Valsalva aneurysm is facilitated by echocardiography, contrast aortography, and more recently, magnetic resonance imaging. Surgical repair is generally required for ruptured aneurysms; unruptured aneurysms encroaching on nearby structures, causing myocardial ischemia, or having the potential to rupture and warrant repair.

\section{Case report}

A 24yrs old pleasant gentleman presented with history of gradually progressive shortness of breath and palpitation on exertion of 5 months duration. He also had history of 2 syncope 6 months prior. On examination he had wide pulse pressure with presence of peripheral aortic run off. Cardiac examination revealed displaced cardiac apex laterally with hyperdynamic apex beat, grade III early diastolic murmur over the aortic area. Rest of the examination was normal. Electrocardiogram revealed LBBB with first degree Atrio-Ventricular block with occasional ventricular premature beats. Chest X-Ray showed prominent bronchovascular markings. 2D echocardiogram revealed aneurysmal dilatation of right sinus of valsalva (SOV) which was burrowing and dissecting into interventricular septum (IVS) echo free space as shown in Figure 1. LV was dilated with LVEDD of $5.4 \mathrm{~cm}$ and LVESD of $4.0 \mathrm{~cm}$ and reduced LV function (LVEF $40 \%$ ). Aortic annulus was dilated $(3 \mathrm{~cm})$ and ascending aorta was of normal size. Aortic valve was tricuspid but deformed and calcified with evidence of severe aortic regurgitation. There was no communication of right sinus of Valsalva with any of the chambers. RA and RV were of normal size. TEE revealed same findings as in 2D echo, but both the right and left sinus of valsalva were seen dilated with the right coronary cusp (RCC) seen dissecting into the IVS with a nodular calcification in the vicinity of AoV as shown in Figure 2. Aortogram findings were also compatible with the 2D echo and TEE findings, which showed dilated right SOV without any evidence of leak into any of the chambers. Thereafter patient was taken up for aortic valve replacement along with repair and closure of the right sinus of valsalva aneurysm with Dacron patch. Intraoperatively there was a dilated right SOV with a cavity which led to the dissected IVS. The cavity was filled with calcium deposits and mural clots. The aortic side of the aneurysm was closed with Dacron patch, preserving the conduction system and avoiding the right coronary ostium. Post operative echo after 3 months showed completely healed and thrombosed aneurysmal sac of right SOV within the IVS as shown in Figure 3. There was no LV dilation ( $5 \mathrm{cms}$ ) with normal LV functions with normal functioning of the aortic prosthesis.

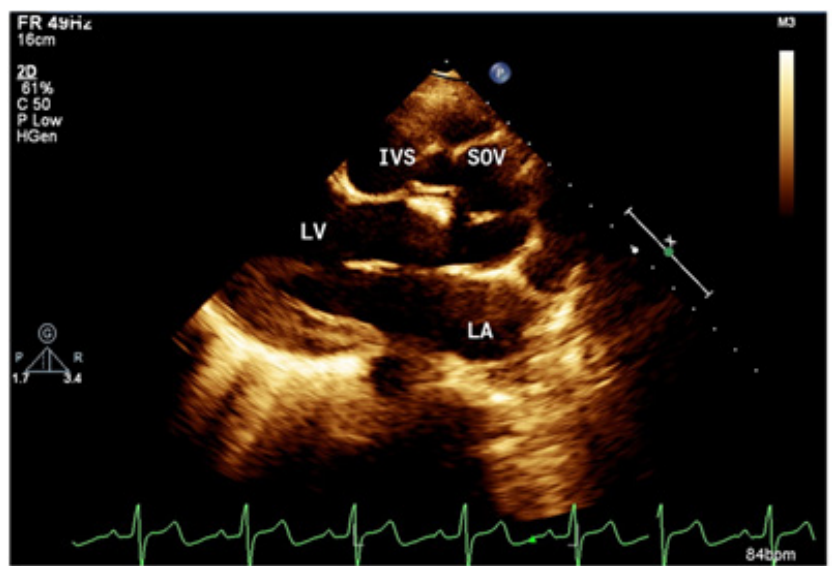

Figure I Aneurysmal dilatation of right Sinus of Valsalva (SOV) burrowing and dissecting into interventricular septum (IVS) - echo free space. 


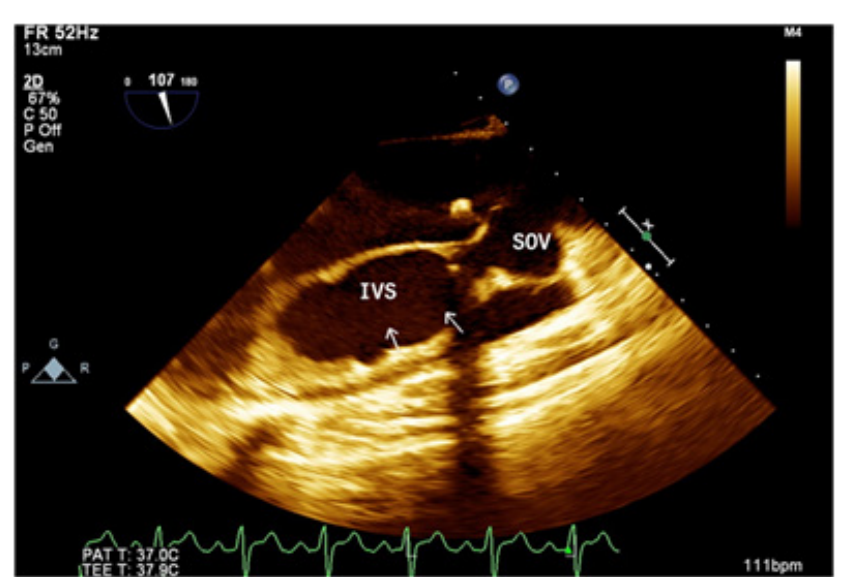

Figure 2 Transesophageal echo: RCC dissecting into the IVS with a nodular calcification in the vicinity of AoV.

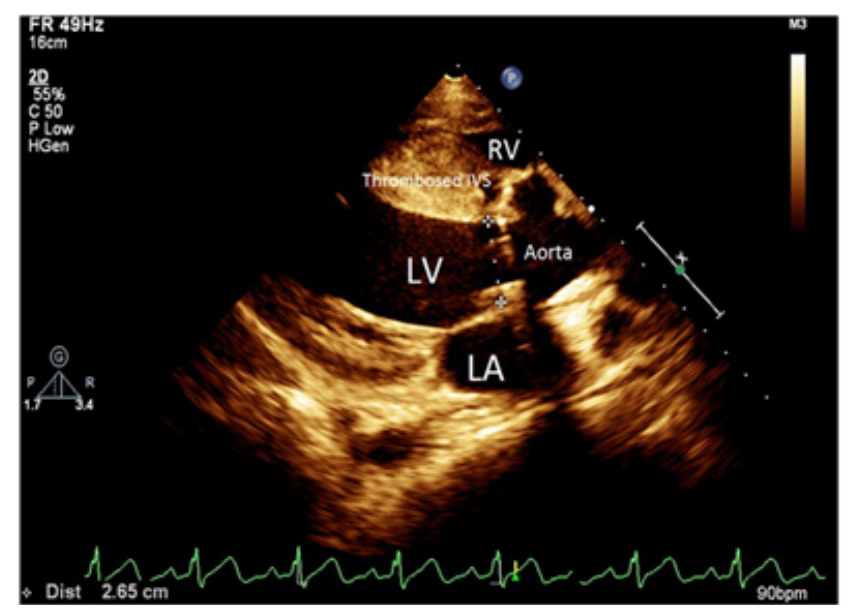

Figure 3 Healed and thrombosed aneurysmal sac of right SOV within the IVS.

\section{Discussion}

A sinus of valsalva aneurysm is a rare cardiac anomaly ranging from 0.1 to $3.5 \%$ of all congenital heart defects and $0.14 \%$ of all open heart procedures. ${ }^{6}$ Sinus of valsalva aneurysms may be congenital or acquired and about $20 \%$ of congenital aneurysms may be unruptured and are discovered at necropsy or surgery. ${ }^{7}$ SVA is more prevalent in Asian surgical series (0.46-3.5\%) and correlates with more supracristal ventricular septal defects $(\sim 60 \%) .{ }^{8}$ Congenital aneurysms occur more frequently in males than females (4:1), more commonly from right coronary sinus, and take an entirely intracardiac course. They extend in diverticular fashion into cardiac cavity and rupture frequently into right heart chamber forming an aorto-cardiac fistula and rarely in some patients aneurysm may dissect into interventricular septum. ${ }^{9}$ LBBB and AV blocks (trifascicular block with syncope in our case) can occur likely due to compression of AV bundle within the IVS. ${ }^{10}$ Congenital Sinus of Valsalva aneurysms that don't communicate with a cardiac chamber remain silent usually until perforation or infective endocarditis. ${ }^{10}$ The frequency of rupture is unknown, but in males rupture usually occurs after puberty and before the age of 30years. ${ }^{11}$ In the past it was thought to be fatal, but in the present era, open heart surgery outcomes have improved, so appropriate timely diagnosis is important. Morphological changes can occur in aortic valve; probably they are congenital or may be due to constant trauma to the aortic leaflets due to AR jet. ${ }^{12}$ Transthoracic echocardiography examination including Doppler and color flow imaging is the diagnostic modality help in making a diagnosis. MRI may help in revealing additional morphological features. ${ }^{13}$ Other coronary anomalies and rupture of aneurysm can also be confirmed by retrograde aortography.

\section{Acknowledgments}

None.

\section{Conflicts of interest}

Author declares there are no conflicts of interest.

\section{Funding}

None.

\section{References}

1. Fishbein MC, Obma R, Roberts WC. Unrupturedsinus of Valsalva aneurysm. Am J Cardiol. 1975;35(6):918.

2. Ring WS. Congenital Heart Surgery Nomenclature and Database Project: Aortic Aneurysm, Sinus of Valsalva Aneurysm, and Aortic Dissection. Ann Thorac Surg. 2000; 69(4 Suppl):S147-S163.

3. Feldman DN, Roman MJ . Aneurysms of the sinuses of Valsalva. Cardiology. 2006;106(2):73-81.

4. Batiste C, Bansal RC, Razzouk AJ. Echocardiographic features of an unruptured mycotic aneurysm of the right aortic sinus of Valsalva. $J \mathrm{Am}$ Soc Echocardiogr. 2004;17(5):474-477.

5. Ott DA. Aneurysm of the sinus of Valsalva. Semin Thorac Cardiovasc Surg Pediatr Card Surg Annu . 2006;165-176.

6. Gonzalez JB, Koul S, Sawardekar U, et al. Images in cardiovascular medicine. Sinus of Valsalva aneurysms: a unique case of giant aneurysms involving all 3 sinuses. Circulation. 2008;117(15):e308-311.

7. Chu SH, Hung CR, How SS, et al. Ruptured aneurysms of the sinus of Valsalva in Oriental patients. J Thorac Cardiovasc Surg. 1990;99(2):288-298.

8. Chalmers JA, Grahaim TR, Magee PG. A congenital aneursym of the non-coronary sinus Of Vasalva extending into the wall of the left ventricle. Int J Cardiol . 1989;24:379-381.

9. Onat A, Ersanli 0, Kanuni A, et al. Congenital aortic sinus aneurysms with particular reference to dissection of the interventricular septum. Am Heart J . 1966;72:158-164.

10. Ahmad RA. Sturmian S, Waitson RD. Unruptured aneurysm of the sinus of VaIsalva presenting with isolated heart block: echocardiographic diagnosis ancl successful surgical repair. B3r Heart J. 1989;61:375-377.

11. Friedman WF. Congenital heart disease in infancy ancl chiilclhlocl. In: Braunw-ald E. Heart disease: a textbook of cardiovascular medicine. (4th edn), WB Saunders, Philadelplhia, USA. 1992. p.887-965.

12. D Metras, A O Coulibaly, K Ouattara. Calcified unruptured aneurysm of sinus of Valsalva with complete heart block and aortic regurgitation. Successful repair in one case. Br Heart J . 1982;48(5):507-509.

13. Goldberg N, Zisbrod Z, Kipperman R, et al. Congenital aneurysm of the left coronary sinus and left main coronary artery with fistulous communication to the right atrium in pregnancy. $\mathrm{J}$ Am Soc Echocardiogr. 1990;3: 125-130. 